\title{
Calculating Water Wavelength Using Dispersion Relation and Approximation
}

Chantelle Lee

University of South Florida

Advisors:

Arcadii Grinshpan, Mathematics and Statistics

Ping Wang, School of Geosciences

Problem Suggested By: Ping Wang

Follow this and additional works at: https://digitalcommons.usf.edu/ujmm

Part of the Mathematics Commons

UJMM is an open access journal, free to authors and readers, and relies on your support:

Donate Now

\section{Recommended Citation}

Lee, Chantelle (2019) "Calculating Water Wavelength Using Dispersion Relation and Approximation," Undergraduate Journal of Mathematical Modeling: One + Two: Vol. 9: Iss. 2, Article 3.

DOI: https://doi.org/10.5038/2326-3652.9.2.4902

Available at: https://digitalcommons.usf.edu/ujmm/vol9/iss $2 / 3$ 


\title{
Calculating Water Wavelength Using Dispersion Relation and Approximation
}

\author{
Abstract \\ The dispersion relation equation is used to directly compute wave number and wave length to \\ compliment water wave pressure sensor readings. Waves are measured to help coastal engineering to \\ better mitigate coastal infrastructures. Various given wave periods and water depths are used to show \\ how the dispersion relation equation could compute wave length. Also, shallow and deep-water \\ approximations are formulated from the dispersion relation equation and the results are compared.
}

\section{Keywords}

wave length, dispersion relation equation, shallow and deep-water approximation, gravitational waves

Creative Commons License

(c) (i) (9)

This work is licensed under a Creative Commons Attribution-Noncommercial-Share Alike 4.0 License. 


\section{PROBLEM STATEMENT}

Determine wavelength of oceanic water using the dispersion relation equation and compare the results with computed shallow and deep-water approximations.

\section{MOTIVATION}

Waves are an integral part of human civilization as the majority of large cities are located in coastal environments. The energy produced by waves manipulates sea sediments, beach topography, and pressure of structures. [Wave Measurement] When waves are very large it can cause safety concerns for public structures and citizens cohabitating along coastal environments. The measurement of waves is important because it ensures precautions are taken for public safety and city planning. Waves can be produced via a plethora of reasons including gravitational pull, landslides, and tectonic plate movement, but the majority of waves are formed by wind currents. [Wave Measurement]

The importance of wave analyzation when constructing coastal structures is evident in the collapsing of the Puerto Colombia Pier in March 2009. [Ortiz, 2014] Models are able to reconstruct wave patterns from hurricanes and cold-front that caused the destruction of the pier. Buoys are used to record data of wave measurements, i.e. its height, direction, and period using model software. [Ortiz, 2014] This is an advanced way of calculating these variables.

George B. Airy correctly formulated the Airy theory or linear wave theory of gravitational waves that are dependent on wave height, length, and period. [McLellan, 1965] A dispersion relation equation can be used to calculate wave number and wave length. The wave number and wave length are manually or virtually computed because these variables cannot be 
directly measured by wave pressure sensors. [Wang and Stone, 2005] Proper calculation of wave length can be used to better engineer coastal infrastructure to reduce disasters like in Columbia.

\section{MATHEMATICAL DESCRIPTION AND SOLUTION APPROACH}

The dispersion relation equation used to compute wave length is dependent on gravitational acceleration, wave period, and water depth. Wave length is the distance between two consecutive wave crests or troughs. Gravitational acceleration is a constant of $9.8 \mathrm{~m} / \mathrm{s}^{2}$. Wave period is the time it takes for two consecutive crests to pass a certain point. Water depth is the depth of a wave below the water surface. [Wang and Stone, 2015]

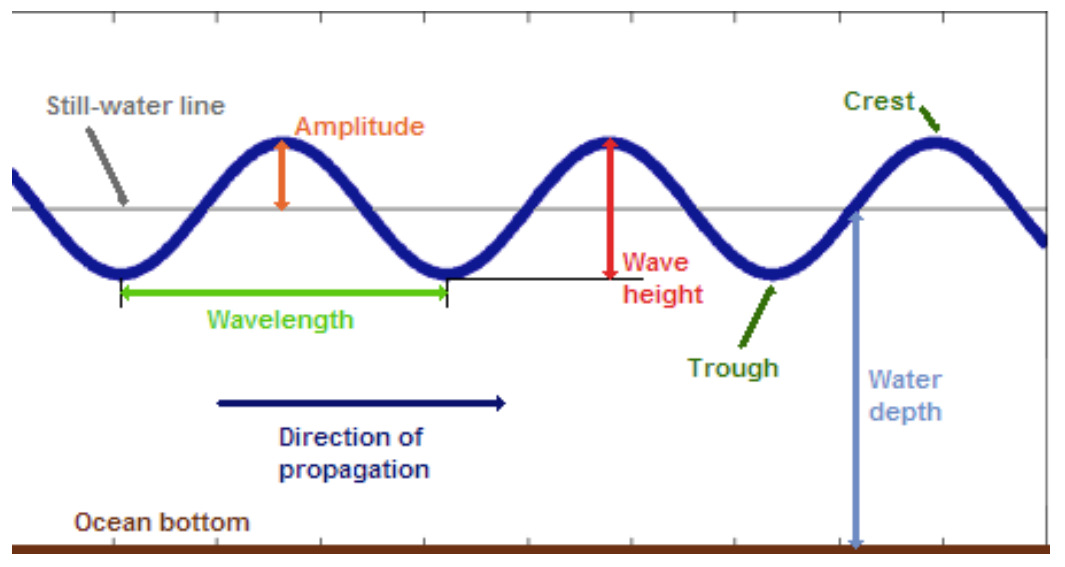

Figure 1. CDIP Document Index Waves

According to linear wave theory, wave length and period are dependent on the dispersion relation equation as

$$
L=\frac{g}{2 \pi} T^{2} \tanh \frac{2 \pi h}{L} .
$$


In equation $1, g$ is the gravitational acceleration constant, $T$ is the wave period, and $h$ is the water depth. The tangent hyperbolic function is used to represent the ratio of hyperbolic sine and hyperbolic cosine. After combining all the constants, the equation can then be represented as

$$
L=1.56 T^{2} \tanh \frac{6.28 h}{L}
$$

When given wave period and depth via wave sensors, one can compute the wave length, $L$, and wave number $(k=6.28 / L)$. Numbers for variables from hereon are hypothetical to give examples of how the dispersion relation can be used to derive $L$ and $k$. Excel worksheet formulation is used to ease the trial and error process of computing these variables.

Six trials are conducted to calculate the wavelength using equation 2: three shallow and three deep water calculations. After computing the results, shallow and deep-water approximations are derived from equation 1. First, deep-water approximation is computed with the assumption that water depth is very large which can be illustrated as:

$$
h \gg L=>x=\frac{2 \pi h}{L} \text { is very large. }
$$

The tangent hyperbolic factor in (1), namely:

$$
\tanh x=\frac{\sinh x}{\cosh x}, \text { where } x=\frac{2 \pi h}{L}
$$

can be replaced with its exponential description 


$$
\tanh x=\frac{\frac{e^{x}-e^{-x}}{2}}{\frac{e^{x}+e^{-x}}{2}}=\frac{e^{2 x}-1}{e^{2 x}+1}
$$

As mentioned above, for the deep-water approximation to work, $x$ is assumed to be very large. If $x$ is very large, then $e^{2 x}$ is very large. Hence we can use the following approximation:

$$
e^{2 x}-1 \approx e^{2 x}+1
$$

Therefore, the tangent hyperbolic function of $x$ is approximately equal to 1 , so the deep-water approximation can be represented as

$$
L=\frac{g}{2 \pi} T^{2}=1.56 T^{2}
$$

Equation 3 is used to validate deep-water results from equation 1 using all the known variables.

Shallow water approximation is also derived from equation 1 . The very common Taylor series at zero of the exponential function $e^{x}$ is used to derive the equation for shallow water approximation: $e^{x}=1+\frac{x}{1 !}+\frac{x^{2}}{2 !}+\cdots \approx 1+x$, where $x$ is small.

Hence $e^{2 x} \approx 1+2 x$ for small $x$ and 


$$
\tanh x \approx \frac{1+2 x-1}{1+2 x+1}=\frac{2 x}{2(x+1)}=\frac{x}{x+1} ; x=\frac{2 \pi h}{L}
$$

To properly calculate the shallow water approximation, $x$ is assumed to be very small. Plugging in the above computation into equation 1 will result in the approximation of

$$
\begin{aligned}
& L=\frac{g}{2 \pi} T^{2}\left(\frac{\frac{2 \pi h}{L}}{\frac{2 \pi h}{L}+1}\right) \approx \frac{g}{z \pi} T^{2}\left(\frac{z \pi h}{L}\right) \\
& \\
&=>L \approx T \sqrt{g h} .
\end{aligned}
$$

Now that approximation equations have been derived, the known variables can be plugged in to find the approximations that correspond with equation 1. For example, using equation 1 with variables $T=7, h=3$, and $\mathrm{L} \approx 36.93$ it can be represented as,

$$
36.93 m=1.56 \times\left(7^{2}\right) \times \tanh \left(\frac{6.28 \times 3}{36.93}\right) \approx 35.932 m .
$$

The dispersion relation equation can be used to get the wavelength value within $<1 \mathrm{~m}$. Using the shallow water approximation gets an accurate value within $1 \mathrm{~m}$. This approximation computation is as follows:

$$
L=T \sqrt{g h}=7 \sqrt{9.8 \times 3}=37.955 m
$$


Besides the fact that using the approximation equations is more time saving, the approximation seems to be more straightforward if someone does not have the appropriate resources.

The Dispersion Relation equation seems to be even more accurate with deep water calculations due to the very large water depth which makes hyperbolic tangent to be very close to 1. For example, with variables $T=7, h=350$, and $L \approx 76.42$ it is computed as,

$$
76.42 m=1.56 \times\left(7^{2}\right) \tanh \left(\frac{6.28 \times 350}{76.42}\right) \approx 76.44 m
$$

The deep-water approximation for this wave period and water depth is

$$
L=\frac{g}{2 \pi} T^{2}=1.56 T^{2}=1.56\left(7^{2}\right) \approx 76.44 m
$$

Deep-water calculations using equation 1 and the approximations are very accurate within a tenth of a difference.

\section{DISCUSSION}

Equation 1 or 2 is used to find wave lengths at different wave periods and water depths. From the Dispersion Relation equation, shallow and deep-water approximations are specifically derived for shallow and deep-water values. Equation 2 is tedious to use due to the same unknown value $(L)$ being on either side of the equation. It is best to use an Excel worksheet to compute the calculations for each problem set. Table 1 showcases the values that are computed via Excel. 


\begin{tabular}{|c|c|c|c|c|}
\hline Trials & $\mathrm{T}(\mathrm{s})$ & $\begin{array}{c}\text { Depth } \\
\text { Description }\end{array}$ & $\mathrm{h}(\mathrm{m})$ & $\mathrm{L}(\mathrm{m})$ \\
\hline 1 & 7 & Shallow & 3 & 36.91 \\
\hline 2 & 10 & Shallow & 3 & 53.22 \\
\hline 3 & 10 & Shallow & 5 & 68.15 \\
\hline 4 & 7 & Deep & 25 & 74.45 \\
\hline 5 & 10 & Deep & 300 & 76.42 \\
\hline 6 & 7 & Deep & 350 & 155.98 \\
\hline
\end{tabular}

Table 1. Computed wavelength using Dispersion Relation

Wave length $(L)$ is highly dependent on wave period $(T)$ because the faster a wave passes a successive point, the shorter the wave length is. This can be seen in Trials 1 and 2 that share the same wave depth $(h)$, but different wave period and therefore different wave lengths. The difference in water depth is also irrelevant in Trials 4 and 5. In Trial 4, water depth is shorter and period is quicker, but still shares a similar wave length as in Trial 5.

\begin{tabular}{|c|c|c|c|c|}
\hline Trials & $\mathrm{T}(\mathrm{s})$ & $\begin{array}{c}\text { Depth } \\
\text { Description }\end{array}$ & $\mathrm{h}(\mathrm{m})$ & $\begin{array}{c}\text { L approximation } \\
(\mathrm{m})\end{array}$ \\
\hline 1 & 7 & Shallow & 3 & 37.96 \\
\hline 2 & 10 & Shallow & 3 & 54.22 \\
\hline 3 & 10 & Shallow & 5 & 155.97 \\
\hline 4 & 7 & Deep & 25 & 70.00 \\
\hline 5 & 10 & Deep & 300 & 76.43 \\
\hline 6 & 7 & Deep & 350 & 155.97 \\
\hline
\end{tabular}

Table 2. Shallow and Deep-Water Approximation Wave Lengths 
The shallow and deep-water approximations for these values are as shown in Table 2. The approximations are all within $<2 m$. This high difference of values can be due to equation 2 being hard to use to compute wave length.

As previously discussed, the shallow and deep-water approximations are more ideal to use if time and resources are not available. No doubt, there is highly sophisticated software that can more accurately use Equation 2. Figure 1 further illustrates how close the computations and approximations are to each other.

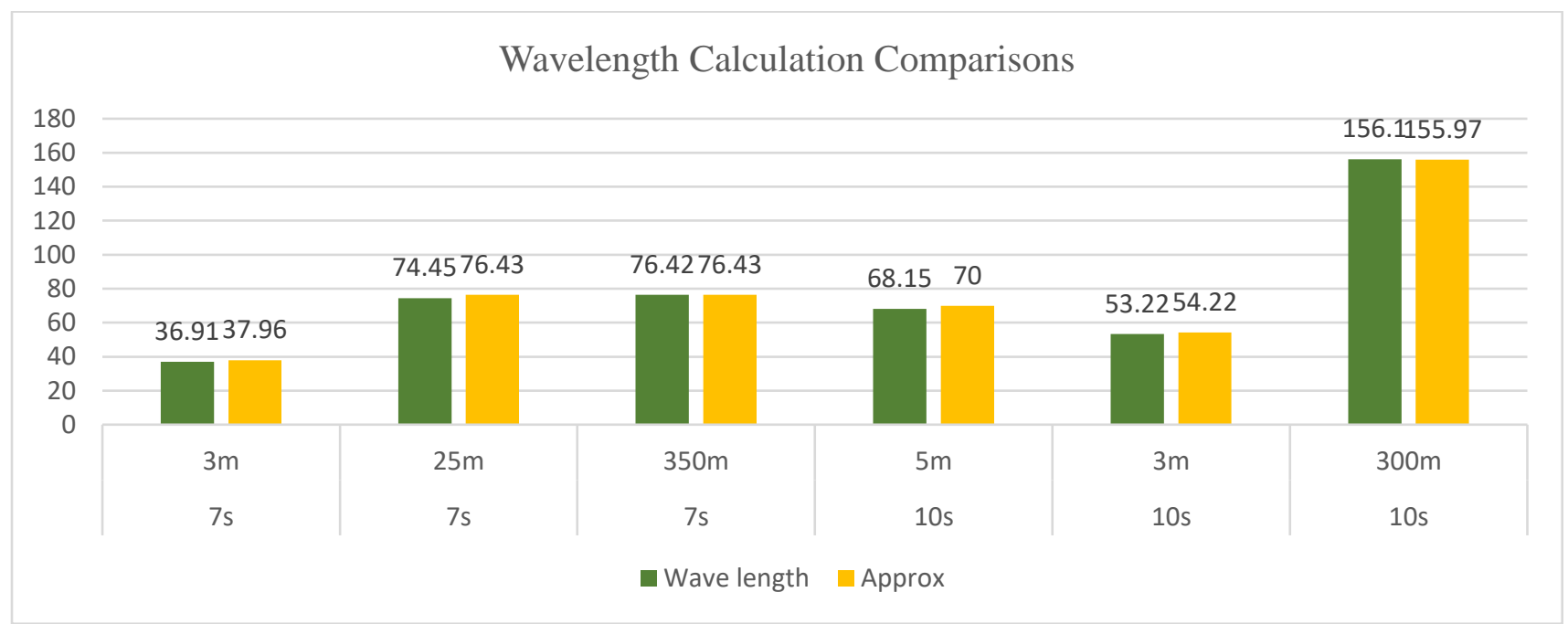

Figure 1. Wavelength Calculation Comparisons

Using the dispersion relation equation allows for the computation of wave length for the known wave periods and water depths. The wave length is computed using Excel spreadsheet to help with the timeliness of the project. After calculating the wave length using dispersion relation, the approximations are derived from the equation for shallow and deep-water values. The approximations are within $<2 m$, with deep-water approximations being highly accurate. The results are as expected as the approximations are derived assuming variables to be either very small or very large. 


\section{CONCLUSION AND RECOMMENDATIONS}

In conclusion, the dispersion relation equation can be used to find wavelength with the

known variables of water depth and wave period. Shallow and deep-water approximations can be derived from the dispersion relation equation to compute characteristically shallow and deepwater values. Deep-water approximations are more accurate than shallow water ones due to hyperbolic tangents being approximately 1 at higher values. The deep-water approximation is reliant only on wave period, while the shallow-water approximation is reliant on wave period and water depth.

The wavelength and wave numbers are calculated using the dispersion relation equation because those values cannot be measured directly from wave pressure sensors. Wave measurements are used primarily by coastal engineers to create reliable coastal infrastructure. This is important because most large cities are along the coast. The tragic incident in Columbia should not occur again and can very well be prevented using wave models and computations.

A recommendation that would have made this project easier is having a better understanding of formulas within Excel worksheets. The more educated someone is in Excel, the easier and less time consuming it would be to calculate wave length. 


\section{NOMENCLATURE}

\begin{tabular}{|c|c|c|}
\hline Symbol & Description & Units \\
\hline$L$ & Wavelength & $9.8 \mathrm{~m} / \mathrm{s}^{2}$ \\
\hline$g$ & Gravitation Acceleration & $\mathrm{s}$ \\
\hline$T$ & Wave Period & $\mathrm{m}$ \\
\hline$h$ & Water Depth & $\mathrm{m}^{-1}$ \\
\hline
\end{tabular}

Table 3. Nomenclature 


\section{REFERENCES}

McLellan, Hugh J. Elements of Physical Oceanography: Pergamon International Library of Science, Technology, Engineering and Social Studies, Elsevier Science \& Technology, 1965. pp. 92 - 106

Ortiz, Juan C., Salcedo, Bladimir, and Otero, Luis J. "Investigating the Collapse of the Puerto Colombia Pier (Colombian Caribbean Coast) in March 2009: Methodology for the Reconstruction of Extreme Events and the Evaluation of Their Impact on the Coastal Infrastructure." Journal of Coastal Research, vol. 30, no. 2, Mar. 2014, pp. 291-300.

The Coastal Data Information Program. "Wave Measurement." CDIP Documents Index Waves, cdip.ucsd.edu/?nav=documents\&sub=index\&xitem=waves.

Wang, Ping, and Gregory W. Stone. "Nearshore Wave Measurement." Encyclopedia of Coastal Science, edited by Maurice L. Schwartz, Springer, 2005, pp. 702-705. 